An update on pelvic inflammatory disease. Ross JDC. Sex Transm Inf 2002; 78: 18-19

The difficulties in clinically diagnosing pelvic inflammatory disease (PID), new diagnostic advances and the relation ship with intrauterine devices (IUDs) are reviewed in this article, which is well worth reading. The sensitivity and specificity of clinical diagnosis is poor with rates of $62 \%$ and $77 \%$, respectively, even with logistic regression. Key predictors are gonococcal or chlamydial infection and an elevated temperature with a high white cell count. Transvaginal ultrasound combined with 'power Doppler' seems promising, as it appears capable of measuring the hyperaemia of tubal inflammation with positive and negative predictive values of $91 \%$ and $100 \%$, respectively. It seems most useful in milder disease but larger studies are needed.

Meta-analysis has identified a relative risk for symptomatic PID of 3.3 in women with IUDs. However, many studies used inappropriate control groups and sexual behaviour was not controlled for. Even if the relative risk of PID is higher in IUD users, the absolute risk remains low and is of the order of 1:1000. Most of the excess PID risk appears limited to the first few weeks after IUD insertion and therefore a major determinant is the prevalence of chlamydial and gonococcal infection. The effectiveness of antibiotic prophylaxis before insertion of an IUD is unproven.

Screening for sexually transmitted infections and rapid empirical use of effective antibiotics remain the cornerstone of PID control.

Reviewed by Elizabeth Carlin, FRCP, DFFP Consultant in Genitourinary Medicine, Nottingham City Hospital NHS Trust, Nottingham, UK
Does HRT double the risk of dementia? NHS Centre for Reviews and Dissemination. Dare Abstract Report 29.5.2003. National Electronic Library of Medicine www.nelm.nhs.uk

A DARE abstract looked at the evidence behind the headlines about the relationship between hormone replacement therapy (HRT) and the risk of dementia. The evidence was generated from the Women's Health Initiative (WHI) Memory Study - a subset of the WHI Study. The objective of the study was to assess the effect of oestrogen and progestogen HRT on the incidence of dementia in postmenopausal women aged 65 years or above. The study was well designed randomised, placebo-controlled, double-blinded, and well powered to justify the conclusion that HRT doubles the risk of dementia. This refers to an absolute increase of 23 cases per 10000 women per year. These findings do not necessarily relate to the majority of women who take HRT at a younger age for menopausa symptoms. The preparation of HRT used in the study is not available in the UK.

Reviewed by P S Arunakumari, MD, MRCOG Specialist Registrar in Obstetrics and Gynaecology, Norfolk and Norwich University Hospital, Norwich, UK

Quality of life following early medical or surgical abortion. Westhoff C, Picardo L, Morrow E. Contraception 2003; 67: 41-47

This study took place in a private practice setting in New York. Women who were having an abortion before 9 weeks' gestation were invited to enrol in the study. The researchers went through validated 30-item questionnaire on three occasions with the 97 patients who were willing to participate. The first questionnaire was filled in at the end of the first consultation, the second one week later at the time of the termination, and the third by telephone after a month. The scores on the questionnaire were compared at the three points of time.

At the baseline assessment, patients reported numerous symptoms and functional impairments. At 1 week, except for worse scores for physical function and pain, most measures showed some improvement. By 1 month, the scores for each type of function and symptom had improved. Improvements were marked in emotional function, appetite and global health. Those who opted for a surgical termination (42) had more symptoms and a higher level of impairment of function at the baseline assessment than those who chose a medical termination (55). The authors speculated that those who were most distressed wished for a speedy resolution of their problem and chose surgical termination. However, by the final assessment there were no obvious differences.

It would be risky to generalise from these results to other settings. Women seeking abortion at private facilities may be unlike those attending publicly funded services. Those who volunteer to complete questionnaires may differ from those who decline - in particular, those who are most distressed or most uncertain about their actions may decline. However, this survey does add to a growing literature that some women may have a better quality of life, and feel relief rather than distress, once the difficulties of facing an unwanted pregnancy are overcome.

Reviewed by Gill Wakley, MD, MFFP

Visiting Professor in Primary Care Development. Staffordshire University and Freelance General Practitioner and Writer, Abergavenny, UK

\section{WEBSITE REVIEWS}

\section{Websites for women with abnormal smears}

A recent research paper suggests up to $40 \%$ of women will have abnormal cytology at some time in their lives. ${ }^{1}$ An abnormal smear result can be extremely distressing and it is useful to be able to recommend some good sites.

www.womens-health.co.uk is an excellent website with sensible information in all areas of obstetrics and gynaecology for the general public. Edited by Danny Tucker, Clinical Lecturer at Oxford University, this site reliably provides helpful guidance for women in the UK health care context. On abnormal smears, the site explains the implications of different grades of dyskaryosis and is sensibly reassuring. It manages to be detailed but not confusing. There is also a good patient-orientated description of what may happen at a colposcopy clinic visit.

www.cancerbacup.org.uk is a fully comprehensive website on understanding cancer of the cervix. This is one of the few websites that addresses the psychosocial issues related to cervical cancer as well as its influence on sex life and fertility.

www.bbc.co.uk/health/features/ cervicalsmear provides a brief overview of cervical cancer testing. The format is userfriendly and the text boxes provide a quick glance at the statistics.

www.medinfo.co.uk emphasises that abnormal results are not always worrying results
- for example, thrush may be present. It provides reassurance in that 'the majority settle back to normal with little or no action'.

The NHS Cervical Screening Programme maintains a good, comprehensive and frequently updated website at www.cancerscreening.nhs. uk/cervical. The advice and information is brief, easily understood and generally reassuring. The section describing treatment for abnormal smear is good, although the mention of hysterectomy may cause undue alarm. There are also rapid responses to any headlines about cervical smears or cancer.

Reference

Raffle A, Alden B, Quinn M, et al. Outcomes of screenin to prevent cancer: analysis of cumulative incidence of cervical abnormality and modelling of cases and death prevented. BMJ 2003; 326: 901-904.

Reviewed by Kate Weaver, MB ChB, MFFP Staff Grade Doctor in Reproductive Healthcare Dean Terrace Family Planning and Well Woman Clinic, Edinburgh, UK

and P S Arunakumari, MD, MRCOG

Specialist Registrar in Obstetrics and Gynaecology, Norfolk and Norwich University Hospital, Norwich, UK

\section{Connexions Direct}

Connexions Direct is a new service for young people aged 13-19 years that offers quick acces to information and advice on a wide range of topics from careers to self-esteem through one easy to use website.

Young people can contact a Connexions Direct adviser by telephone, webchat, e-mail or text message. If young people want to arrange to speak to a local personal adviser face to face, then they can click on the Connexions service button on the home page for details of their local
Connexions partnership. All areas of the country are now covered. The advisers are there to listen, but can offer confidential advice and practical help too. If young people need even more specialist help the advisers know where they can obtain it. Information about services such as Brook or fpa is explained and links to other more focused sites on sexual health or relationships are provided.

All advice is confidential and young people don't have to give a name and address if they don't want to. Information will not be shared with anyone outside the helpline unless advisers think that the person contacting the service or another young person is in danger or at risk of serious harm. If so, only those who need to know will find out and advisers will discuss it with the individual first and offer support. Webchat or email from the website at www.connexions. gov.uk.

\section{Women's sexual health choices}

'Power to choose: young women and sexual health' gives health professionals an overview of sexual health choices available to women. This website developed by the Young Women's Christian Association (YWCA) also includes a summary of existing research about sexual health for women. Several other publications available either to purchase or to download concern women's issues but readers may (like I did) find it a little difficult to find specific information. Good if you just want to browse for ideas, though. Website address: www.ywca-gb.org.uk/ pcpublications.asp.

Reviewed by Gill Wakley, MD, MFFP

Visiting Professor in Primary Care Development Staffordshire University and Freelance General Practitioner and Writer, Abergavenny, UK 